\title{
Estimation of Non-Cartesian Local Structure Tensor Fields
}

\author{
Björn Svensson, Anders Brun, Mats Andersson, and Hans Knutsson \\ Department of Biomedical Engineering, Medical Informatics \\ Center for Medical Image Science and Visualization \\ Linköping University, Sweden \\ $\{$ bjosv, andbr, matsa, knutte\}@.imt.liu.se
}

\begin{abstract}
In medical imaging, signals acquired in non-Cartesian coordinate systems are common. For instance, CT and MRI often produce significantly higher resolution within scan planes, compared to the distance between two adjacent planes. Even oblique sampling occurs, by the use of gantry tilt. In ultrasound imaging, samples are acquired in a polar coordinate system, which implies a spatially varying metric.

In order to produce a geometrically correct image, signals are generally resampled to a Cartesian coordinate system. This paper concerns estimation of local structure tensors directly from the non-Cartesian coordinate system, thus avoiding deteriorated signal and noise characteristics caused by resampling. In many cases processing directly in the warped coordinate system is also less time-consuming.

A geometrically correct tensor must obey certain transformation rules originating from fundamental differential geometry. Subsequently, this fact also affects the tensor estimation. As the local structure tensor is estimated using filters, a change of coordinate system also change the shape of the spatial support of these filters. Implications and limitations brought on by sampling require the filter design criteria to be adapted to the coordinate system.
\end{abstract}

\section{Introduction}

Tensor fields occur frequently in image processing, either as physical measurements for instance diffusion tensors and strain tensors, or as features representing local signal characteristics such as local structure, orientation, junctions, corners and curvature. A common view, is to look upon the tensor as a matrix. For warped coordinate this view is however in conflict with the viewpoint of this paper, i.e. a tensor is a geometric object. Most algorithms for processing or estimating tensor fields from sampled signals in non-Cartesian coordinate systems face the same problem, which can be summarized in three points and is also illustrated by Fig. 1].

- A tensor definition is required obey the transformation rules brought on by differential geometry.

- Any filters used needs to be consistent with this definition, at least if the continuous signal is available. As a consequence the resulting tensor will 

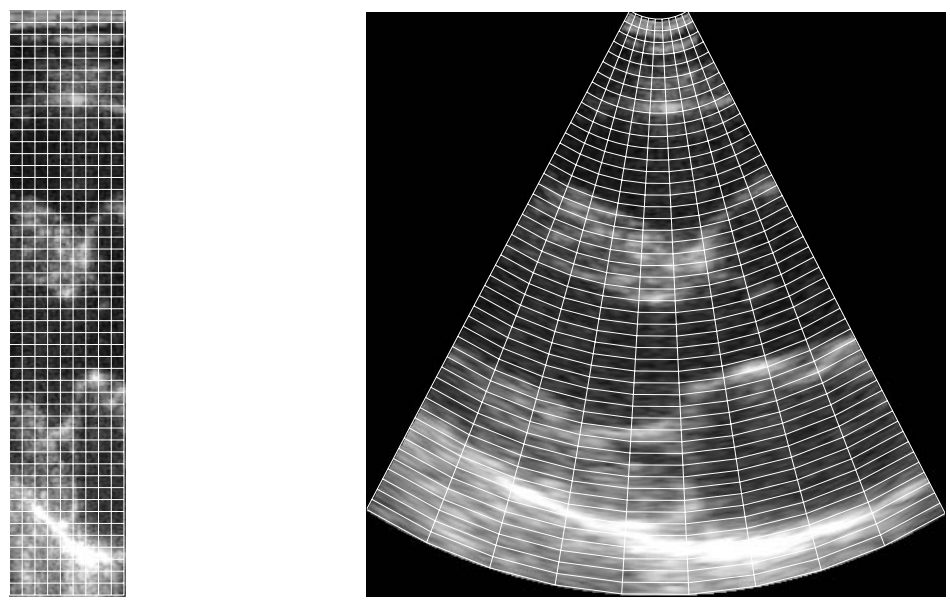

Fig. 1. Ultrasound images is one obvious example of when acquisition is performed in an non-Cartesian coordinate system. Image statistics are often described in the correct geometry, i.e. a Cartesian coordinate system (right) while noise statistics in general are easier to describe and thus also to suppress in the original (polar) coordinate system (left). A filter response is obtained by integrating the signal in a small neighborhood, usually defined in the correct geometry. Carrying out the same calculation in the polar grid requires reshaping the area to integrate.

be geometrically correct even if it is processed or estimated in a warped coordinate system.

- Discrete sampling implies that issues such as aliasing must be considered. Implementation of efficient filters which approximate the ideal behavior is required.

To obtain a true geometric description it is essential that the imaging device provides a signal sampled in a Cartesian coordinate system. In medical imaging this condition is in general not fulfilled. Resampling before computation or processing of the tensor field is the standard approach to compensate for such geometric distortion. However, resampling deteriorates signal and noise characteristics, which significantly complicates estimation or processing of the tensor field.

The concept of representing local image structure with tensors introduced in 1], has proved to be useful for estimating e.g. orientation, velocity, curvature and diffusion. Adaptive anisotropic filtering [2] and motion compensation [3] are examples of algorithms that rely on local structure tensor fields. Estimating local structure for curved manifolds or samples acquired from non-Cartesian coordinate system is a relatively unexplored area of research. Steps in this direction has been taken in 45. In the former, affine filters are used in the framework of adaptive filtering for simultaneous resampling and compensation for geometric distortion. The latter concerns transforming tensor fields in space-time geometry used for motion estimation. 


\section{Tensor Calculus}

To introduce the notation and to emphasize important differences between Cartesian and non-Cartesian tensors, this section gives a brief introduction to tensor calculus. Since this paper concerns local structure tensors, second order tensors are frequently used in the examples.

A contravariant tensor $T \in V \otimes V$ with components $T^{i j}$ is said to be of order $(2,0)$ with two upper indices. A covariant tensor $T \in V^{*} \otimes V^{*}$ of order $(0,2)$ has two lower indices, where $V^{*}$ is the dual space of the vector space $V$. Moreover, a contravariant vector $v$ is a tensor of order $(1,0)$, i.e. an element $v \in V$. Consequently, a covariant vector $w \in V^{*}$ is a tensor of order $(0,1)$. The $d$-dimensional vectors $v$ and $w$ with components $v^{i}, w_{i}$ are expressed in the basis $\frac{\partial}{\partial x^{i}}$ and $d x^{i}$ in Eq. 1 .

$$
v=v^{1} \frac{\partial}{\partial x^{1}}+v^{2} \frac{\partial}{\partial x^{2}}+\cdots+v^{d} \frac{\partial}{\partial x^{d}} \quad w=w_{1} d x^{1}+w_{2} d x^{2}+\cdots+w_{d} x^{d}
$$

In tensor calculus the Einstein summation convention is frequently used to streamline algebraic expressions. It means that indices occurring more than once in an expression are implicitly summed over, which allows a convenient notation, e.g. second order contravariant and covariant tensors $S, T$ can be compactly written as in Eq. 2

$$
S=S^{i j} \frac{\partial}{\partial x^{i}} \frac{\partial}{\partial x^{j}}=\sum_{i, j} S^{i j} \frac{\partial}{\partial x^{i}} \frac{\partial}{\partial x^{j}} \quad T=T_{i j} d x^{i} d x^{j}=\sum_{i, j} T_{i j} d x^{i} d x^{j}
$$

The metric tensor $g$ defines an inner product in $V$, which makes it possible to identify elements in $V^{*}$ with elements in $V$. The metric tensor is a covariant second order tensor, with elements $g_{i j}$. Consequently the metric allows us to move between covariant and contravariant tensors illustrated in Eq. 3 .

$$
g(u, v)=\langle u, v\rangle=g_{i j} u^{i} v^{j} \quad w=g(\cdot, v)=w_{i} d x^{i} \quad \Longrightarrow \quad w_{i}=g_{i j} v^{j}
$$

The metric tensor in the Euclidean space is the Kronecker delta, i.e. $g_{i j}=\delta_{i j}$. Consequently, covariant and contravariant tensors are equivalent in a Cartesian coordinate system. Such tensors are called Cartesian tensors. A change of coordinate system from $x$ to $\tilde{x}$ yields new components expressed in a new basis. Hence, the transformation behavior for a tensor second order contravariant tensor $T$ with components $T^{i j}$ and basis $\frac{\partial}{\partial x^{i}} \frac{\partial}{\partial x^{j}}$ is easily derived. The tensor components $T^{i j}$ transforms to $\tilde{T}^{i j}$ by the chain-rule according to Eq. 4.

$$
T=\tilde{T}^{i j} \frac{\partial}{\partial \tilde{x}^{i}} \frac{\partial}{\partial \tilde{x}^{j}}=T^{i j} \frac{\partial}{\partial x^{i}} \frac{\partial}{\partial x^{j}} \quad \Longrightarrow \quad \tilde{T}^{i j}=\frac{\partial \tilde{x}^{i}}{\partial x^{k}} \frac{\partial \tilde{x}^{j}}{\partial x^{l}} T^{k l}
$$

In the same way a covariant second order tensor $T$ with components $T_{i j}$ transforms to $\tilde{T}_{i j}$ by Eq. 5

$$
T=\tilde{T}_{i j} d \tilde{x}^{i} d \tilde{x}^{j}=T_{i j} d x^{i} d x^{j} \quad \Longrightarrow \quad \tilde{T}_{i j}=\frac{\partial x^{k}}{\partial \tilde{x}^{i}} \frac{\partial x^{l}}{\partial \tilde{x}^{j}} T_{k l}
$$


A uniform stretch of the coordinate system, i.e. the transformation $\tilde{x}=a x$ with $a>1$ causes covariant tensor components $T_{i j}$ to shrink, while the contravariant components $T^{i j}$ grow. Thus, it is of outmost importance to know whether the tensor is contravariant or covariant.

The contraction of a tensor of order $(p, q)$ yields a tensor of order $(p-1, q-1)$, e.g. the trace of $T^{i} j$ in Eq. 6 gives a scalar (a tensor of order 0). Note also that a contraction is only defined for mixed tensors, i.e. tensors with both contravariant and covariant indices.

$$
\operatorname{trace}\left(T^{i}{ }_{j}\right)=T_{i}^{i}=\sum_{i} T_{i}^{i}
$$

Another example of a contraction is the eigenvalue equation tensors in Eq. 7 Note again that the eigenvalue equation is only defined for mixed tensors, i.e. if the tensor is not mixed, eigenvalue decomposition can not be performed without a metric.

$$
T_{j}^{i} v^{j}=g^{i k} T_{k j} v^{j}=\sum_{j, k} g^{i k} T_{k j} v^{j}=\lambda v^{i}
$$

With a metric $g^{i j}$ equal to the identity operator, i.e. a Cartesian coordinate system, the standard eigenvalue equation is obtained. Now consider solving the eigenvalue equation for non-Cartesian tensors. The eigenvalues will be invariant to a change of coordinate system, while the eigenvectors are not.

\section{$3 \quad$ Local Structure Tensor Fields}

An orientation tensor, a covariant second order tensor $T \in V^{*} \otimes V^{*}$, is defined for simple signal neighborhoods $s=f\left(u_{i} x^{i}+\theta\right)$. A simple signal $s$ is a scalar function intrinsically 1-dimensional, i.e. constant in the direction orthogonal to $u$. In most practical applications $V$ is the Euclidean space spanned by its natural basis $\frac{\partial}{\partial x^{i}}$. Given a simple signal, $T$ is defined by Eq. 8 , i.e. the outer product of the signal direction $u$.

$$
T_{i j}=\lambda u_{i} u_{j}, \quad \lambda \geq 0
$$

As opposed to a vector representation, signal orientation can be continuously represented by a tensor. Using vectors, there is no distinction between a signal with direction $-u_{k}$ and a signal in the direction of $u_{k}$. The outer product however yields a representation which maps both $u_{k}$ and $-u_{k}$ to the same geometric object, the orientation tensor [1].

To represent orientation, the tensor must meet two fundamental requirements. Firstly, the tensor norm must be invariant to rotation, i.e. $\|T\|$ is independent of the direction of $u$. Secondly, the tensor must be phase-invariant, i.e. $T$ is independent of any phase-shift $\theta$ of the signal $s$. These partly contradictory requirements are hard to accomplish for sampled signals. Hence, several different approaches how to estimate the tensor has been suggested (e.g. 66/7,8,9,10]). Note that all of them does not claim to meet these requirements. The behavior for non-simple signals are entirely defined by the estimator used. An entire class of 

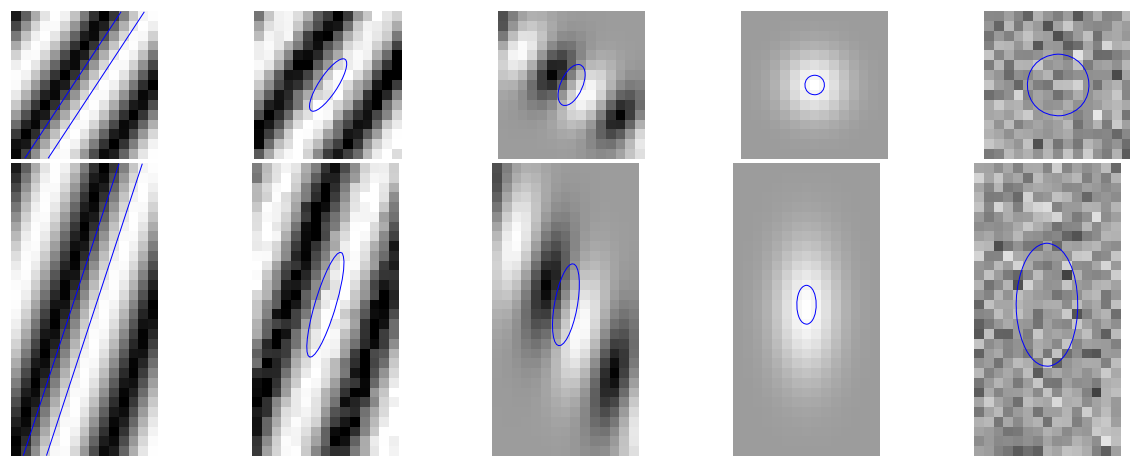

Fig. 2. A few samples of two-dimensional signal neighborhoods. They represent a simple signal (a), a simple signal corrupted with white noise (b), a non-simple signal (c), a signal with no orientation (d), and white noise (e). The patches in the top row are sampled in a Cartesian coordinate system, while the bottom row shows the same signals, sampled more densely in the vertical direction. The tensor glyph $T_{i j} v^{i} v^{j}=1$ is displayed. Note how the glyph is stretched in the same way as the coordinate system, which is a correct transformation behavior.

functions that meets the fundamental requirements of the orientation tensor are derived in [1].

For non-simple signals, the orientation tensor does however carry more information about the local signal neighborhood, like for instance degree of anisotropy and how planar/linear a 3-dimensional neighborhood is. The term local structure tensor in Eq. 9 is a wider interpretation of the orientation tensor, representing local signal neighborhoods that are concentrated to a $k$-dimensional subspace in the Fourier domain, spanned by the orthonormal basis $u_{1}, u_{2}, \cdots, u_{k}$.

$$
T_{i j}=\lambda^{k} u_{i k} u_{j k}, \quad \lambda^{1}, \lambda^{2}, \cdots, \lambda^{k} \geq 0
$$

For displaying purposes, glyphs are a common way to visualize a tensor field. The isometric tensor glyph in Eq. 10 can be used to represent a second order covariant tensor, which is positive semidefinite. Moreover this glyph is invariant to change of coordinate system. The size of the glyph is inversely proportional to $\sqrt{\lambda}$.

$$
T_{i j} v^{i} v^{j}=1
$$

In Fig. 2 a few sample patches are shown in two different coordinate systems. The bottom row is the same continuous signal sampled more densely in the vertical direction, i.e. the top row shows Cartesian tensors while the bottom row shows non-Cartesian tensors, sampled in the coordinate system $\tilde{x}^{2}=a x^{2}$, with $a>1$. Drawing the local structure tensor in this way implies that the local structure tensor can be interpreted as a local image metric, describing how the signal energy measure is locally distributed. The tensor glyph is then the isocurve where this measure is constant. This interpretation presumes a positive semidefinite tensor. 


\section{Tensor Estimation}

In this paper quadrature filters are used to estimate the local structure tensor. Without going through the details, a quadrature filter responses is a phaseinvariant signal energy measure projected on a discrete number of directions $n_{k}$. The projections, i.e. the filter responses $\left\{q_{1}, q_{2}, \cdots, q_{k}\right\}$ (scalar values) for simple signals of single frequencies are given by Eq. 11.

$$
\left|q_{k}\right|=\lambda\left(u_{i} n_{k}^{i}\right)^{2}
$$

A tensor estimate in arbitrary direction is then obtained Eq. 12 by linearly combine the filter responses by the use of a tensors $\left\{M^{1}{ }_{i j}, M^{2}{ }_{i j}, \cdots M^{k}{ }_{i j}\right\}$, where each $M_{i j}$ is dual to the outer product of its corresponding filter direction $n^{i} n^{j}$.

$$
T_{i j}=\left|q_{k}\right| M^{k}{ }_{i j}
$$

A quadrature-based estimate of the local structure tensor is approximately phase-invariant for signals of low bandwidth, i.e. the tensor is insensitive to small shifts of the signal $s$. Since the tensors $T, M$ are all elements in $V^{*} \otimes V^{*}$, a local structure estimate $\tilde{T}_{i j}$ in a non-Cartesian coordinate system is obtained by applying quadrature filters adjusted to the new coordinate system and compute the estimate in Eq. 12 with $\tilde{M}_{i j}$ obtained by transforming $M_{i j}$.

Constructing the tensor in this way is consistent with the tensor definition, if the filter responses $q_{k}$ are invariant to a change of coordinate system. The convolution integral in Eq. 13 reveals that $q\left(\tilde{x}^{k}\right)=q\left(x^{k}\right)$ if the filter is transformed in the same way as the signal, i.e. $h\left(\tilde{x}^{k}\right)=h\left(a^{i}{ }_{k} x^{k}\right)$. This is not at all surprising, since a filter produce a scalar value and can be interpreted as a projection. It is however important to realize that the spatial support $\Omega$ changes, e.g. an isotropic spatial support becomes anisotropic in the warped coordinate system.

$$
q\left(\tilde{x}^{k}\right)=\int_{\tilde{\Omega}} s\left(\tilde{y}^{k}\right) h\left(\tilde{y}^{k}-\tilde{x}^{k}\right) \frac{1}{\left|\operatorname{det}\left(a^{i}{ }_{k}\right)\right|} d \tilde{y}^{k}=\int_{\Omega} s\left(y^{k}\right) h\left(y^{k}-x^{k}\right) d y^{k}=q\left(x^{k}\right)
$$

Since estimators are usually defined in the frequency domain, it is desirable to translate this result to the frequency domain. If $a^{i}{ }_{k}$ is an affine transform the frequency response of the filter $h\left(\tilde{x}^{k}\right)=h\left(a^{i}{ }_{k} x\right)$ relates to the frequency response of $h\left(x^{k}\right)$ by Eq. 14.

$$
H\left(\tilde{u}_{i}\right)=\mathcal{F}\{h(\tilde{x})\}=\int_{\mathbb{R}^{d}} h\left(\tilde{x}^{k}\right) \exp \left(-i \tilde{u}_{k} \tilde{x}^{k}\right) d x^{k}=\left|\operatorname{det}\left(a_{i}{ }^{k}\right)\right| H\left(a^{-1}{ }_{i}{ }^{k} u_{k}\right)
$$

Thus, an estimate consistent with the definition in Eq. 8 is obtained if $\mathcal{F}\{h(\tilde{x})\}=$ $\left|\operatorname{det}\left(a_{i}{ }^{k}\right)\right| H\left(a^{-1}{ }_{i}{ }^{k} u_{k}\right)$. But so far ignored the effect of sampling has been ignored. The limitations brought on by sampling makes it difficult to estimate a tensor, which is strictly consistent with the definition due to aliasing effects and limited resolution. 

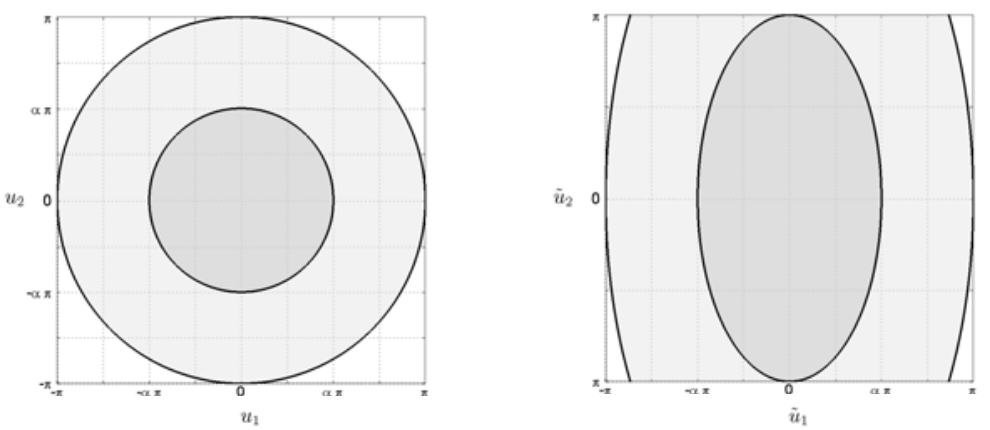

Fig. 3. In a Cartesian coordinate system (left) the filters must suppress the corners of the frequency domain, to ensure that $\|T\|$ does not vary with signal orientation. Aliasing occurs for frequencies which is above the sampling frequency $(\|u\|>\pi)$. Taking fewer samples in the vertical direction implies that aliasing occurs for $\tilde{u}_{2}>\pi$ which correspond to $u_{2}>\alpha \pi$, where $\alpha<1$. To simultaneously avoid aliasing and still get an unbiased tensor estimate in the non-Cartesian coordinate system (right) the filters must suppress frequencies outside $\|u\|<\alpha \pi$ (dark-gray), a region which is isotropic to the left and consequently anisotropic to the right.

\section{$5 \quad$ Filter Design}

For a sampled signal it is enough to study the frequencies contained in $\mathbb{U}=\{u$ : $\left.\left|u_{i}\right| \leq \pi\right\}$, since frequencies outside $\mathbb{U}$ can not be represented and will suffer from aliasing. A change of basis then implies that the sampling distance varies with direction and $\mathbb{U}$ is reshaped. In fact this phenomenon actually occurs already in the Cartesian coordinate system. To get an unbiased estimate of local structure the filters then must suppress the corners of the Fourier domain, i.e. directions in which frequencies above the Nyquist frequency $(\|u\|>\pi)$.

To avoid aliasing, the Shannon sampling theorem states that a signal can be perfectly reconstructed if the signal is band-limited, in this case if the signal is contained in $\mathbb{U}$. Reshaping $\mathbb{U}$ then might imply a limited ability to represent signals as illustrated in Fig. 3. Restricting the transformations to be affine as in the previous section ensures that the Shannon sampling theorem is applicable for the warped coordinate system.

Another important observation is that $h(x)$ and $h(\tilde{x})$ no longer can be the same functions, since samples are acquired at integer positions $z^{k} \in \mathbb{Z}^{d}$ pointing out different locations $z^{i} \frac{\partial}{\partial x^{i}}$ and $z^{i} \frac{\partial}{\partial \tilde{x}^{i}}$ if the coordinate system is changed. It is however possible to choose the values of the filter coefficients $c, \tilde{c}$ at these positions such that $h\left(x^{k}\right)$ and $\tilde{h}\left(\tilde{x}^{k}\right)$ defined by Eq. 15 approximate the same frequency response $H\left(u_{i}\right)$ and $H\left(\tilde{u}_{i}\right)$ derived in the previous section.

$$
h(x)=c_{k} \delta\left(x^{k}-z^{k}\right) \quad \tilde{h}\left(\tilde{x}^{k}\right)=\tilde{c}_{k} \delta\left(\tilde{x}^{k}-z^{k}\right)
$$


Solving the optimization problem in Eq. 16 yields the spatial kernel $\tilde{h}\left(\tilde{x}^{k}\right)$ with the closest fit to the desired frequency response $H\left(\tilde{u}_{i}\right)$.

$$
\min _{c} \varepsilon=\left\|H\left(\tilde{u}_{i}\right)-\mathcal{F}\left\{\tilde{h}\left(\tilde{x}^{k}\right)\right\}\right\|_{W}^{2}+\lambda\left\|\tilde{h}\left(\tilde{x}^{k}\right)\right\|_{w}^{2}
$$

The weighted norm is used to favor a close fit for the most common frequencies and the regularization term is used to favor a small spatial size of the filter kernel 12 .

\section{Experiments}

The following experiment was setup to compare estimation of local structure before resampling to the more common approach where the signal first is resampled using bilinear interpolation to a geometrically correct grid from which the local structure is estimated.

The continuous simple signal $s=\cos \left(u_{i} x^{i}+\theta\right)$ shown in Fig. 2 corrupted with additive white Gaussian noise was sampled in a coordinate system where the vertical axis was shortened a factor 2 , i.e. a patch of size $17 \times 9$ pixels. 60000 signal neighborhoods with 2000 different orientations uniformly distributed and random phase-shift $\theta$ was used in the experiments. Two different local structure tensors were estimated for the neighborhood origin for all patches. One estimated directly in the non-Cartesian coordinate system, one estimated after resampling the patch to a spatial size of $17 \times 17$ pixels, i.e. a Cartesian coordinate system. As a reference a tensor was also estimated from a signal with all samples in the $17 \times 17$ patch available, i.e. a signal with twice the amount of information compared to the two former estimates.

Since $s$ is a simple signal the largest eigenvector $u$ of the tensor should correspond to the signal direction $v$. Measuring the root-mean-square angular error $\Delta \varphi_{r m s}$ reveals the squared deviation between $v$ and $u$. Fig. 4 shows the errormeasures defined by Eq. 17 for the three different tensor estimations. It is also interesting to study how the estimate $u$ varies with the true signal direction $v$ calculating the angular bias $\Delta \varphi$ for all sampled signal orientations.

$$
\Delta \varphi_{r m s}=\cos ^{-1}\left(\sqrt{\frac{1}{n} \sum_{u}\langle u, v\rangle^{2}}\right) \quad \Delta \varphi=\sin ^{-1}\left(\frac{1}{n} \sum_{u}(u \times v) \operatorname{sign}(\langle u, v\rangle)\right)
$$

Estimating the dominant orientation directly in the non-Cartesian system yields a better accuracy compared to the approach where the signal is resampled prior to tensor estimation. A small bias is obtained for noise-free signals due to filter approximation errors. This bias is however very small in comparison to the bias introduced by resampled noise. It should also be mentioned that estimating the non-Cartesian tensor requires no resampling and is roughly 2 times faster due to smaller filters. 

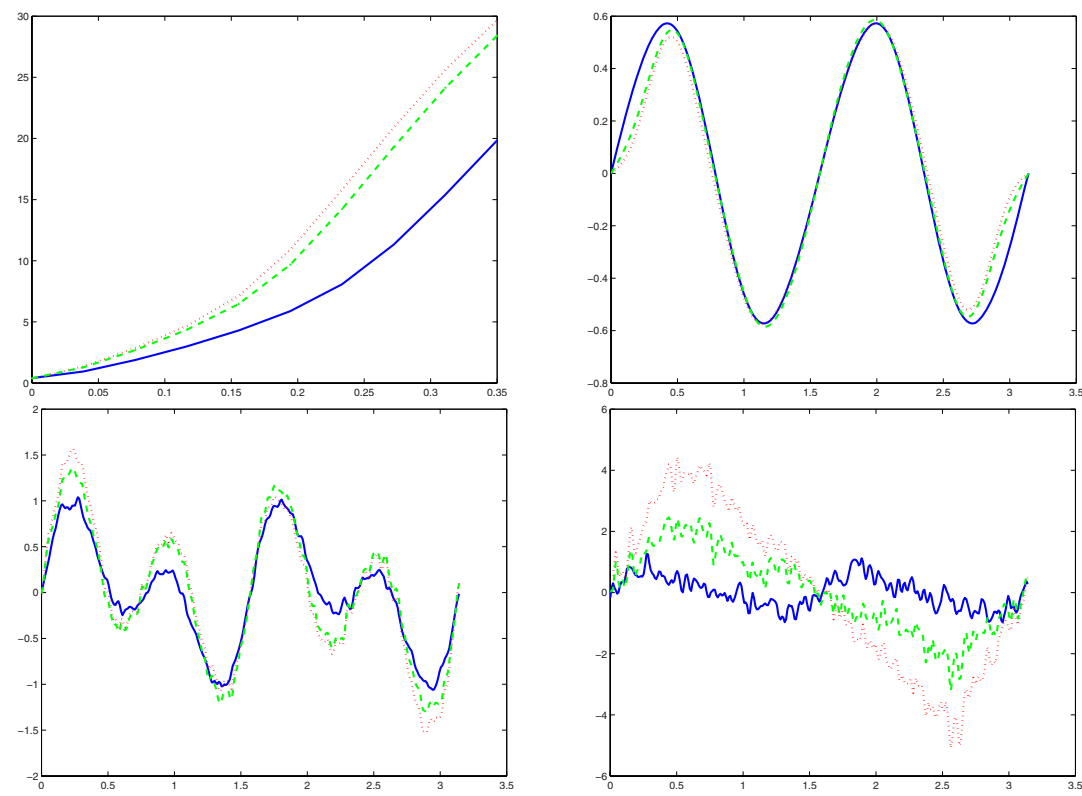

Fig. 4. The angular root mean square error $\Delta \varphi_{r m s}$ in degrees is shown as a function of noise level (upper left), going from a signal-to-noise ratio of $S N R=\infty \mathrm{dB}$ to a signal-to-noise ratio of $S N R=0 \mathrm{~dB}$. Estimating the dominant orientation directly in the non-Cartesian coordinate system (dashed) yields a smaller angular error compared to estimation after resampling (dotted) as the noise level increases. The angular deviation $\Delta \varphi$ as a function of signal orientation $\varphi$ reveals a biased estimate, the mean angular deviation from the true signal varies with signal orientation. For noise-free signal patches (upper right), the deviation is very small and the difference between the non-Cartesian (dashed), resampled(dotted) and the reference is not significant. However for moderate (lower left) and high (lower right) noise levels $(S N R=9.3 \mathrm{~dB}$, $S N R=0 \mathrm{~dB})$ the non-Cartesian estimation shows better performance compared to resampling.

\section{Discussion}

A framework for estimation of local structure directly from sampled signals in warped coordinate systems was presented. This approach can be advantageous in situations where resampling deteriorates signal and noise characteristics. Experiments show that for the test pattern used more accurate estimates can be obtained with less amount of processing. The presented work is also applicable to curved manifolds, provided that they are locally flat, i.e. a relatively low curvature.

Designing filters for estimating non-Cartesian local structure tensors might seem straightforward. However, it becomes more difficult to meet the contradictory demands on locality in both the spatial and the frequency domain, as the 
signal of interest moves closer to the Nyquist frequency it becomes harder to avoid aliasing and at the same time achieve unbiased tensor estimates.

The presented work relies on that the transformation between the coordinate systems can be approximated by an affine transformation, at least locally. This is required for the Shannon sampling theorem to be applicable. However, there are sampling theorems that allow more general transformations as long as the signal band-limitedness is preserved.

\section{References}

1. Knutsson, H.: Representing local structure using tensors. In: The 6th Scandinavian Conference on Image Analysis, Oulu, Finland, June 1989. Report LiTH-ISYI-1019, Computer Vision Laboratory, Linköping University, Sweden pp. 244-251 (1989)

2. Westin, C-F., Knutsson, H., Kikinis, R.: Adaptive image filtering. In: Bankman (ed.) Handbook of Medical Imaging - Processing and Analysis, Academic press, San Diego (2000)

3. Hemmendorff, M.: Motion Estimation and Compensation in Medical Imaging. PhD thesis, Linköping University, Sweden, SE-581 85 Linköping, Sweden, Dissertation No 703 (2001)

4. Westin, C.-F., Richolt, J., Moharir, V., Kikinis, R.: Affine adaptive filtering of CT data. Medical Image Analysis 4(2), 161-172 (2000)

5. Andersson, M., Knutsson, H.: Transformation of local spatio-temporal structure tensor fields. In: IEEE International Conference on Acoustics Speech and Signal Processing (ICASSP), Presented at ICIP 2003 in Barcelona, Spain, (September 20 2003)

6. Knutsson, H.: Filtering and Reconstruction in Image Processing. PhD thesis, Linköping University, Sweden, Diss. No. 88 (1982)

7. Bigün, J., Granlund, G.H., Wiklund, J.: Multidimensional orientation: texture analysis and optical flow. IEEE Transactions on Pattern Analysis and Machine Intelligence, PAMI-13(8) (August 1991)

8. Farnebäck, G.: Orientation estimation based on weighted projection onto quadratic polynomials. In: Girod, B., Greiner, G., Niemann, H., Seidel, H.-P. (eds.), Vision, Modeling, and Visualization 2000: proceedings, pp. 89-96, Saarbrücken (November 2000)

9. Knutsson, H., Andersson, M.: Implications of invariance and uncertainty for local structure analysis filter sets. Signal Processing: Image Communications 20(6), 569$581(2005)$

10. Felsberg, M., Köthe, U.: Get: The connection between monogenic scale-space and gaussian derivatives. In: Kimmel, R., Sochen, N., Weickert, J. (eds.) Scale-Space 2005. LNCS, vol. 3459, pp. 192-203. Springer, Heidelberg (2005)

11. Nordberg, K., Farnebäck, G.: Estimation of orientation tensors for simple signals by means of second-order filters. Signal Processing: Image Communication 20(6), 582-594 (2005)

12. Knutsson, H., Andersson, M., Wiklund, J.: Advanced filter design. In: Proceedings of the 11th Scandinavian Conference on Image Analysis, Greenland, SCIA (June 1999) 